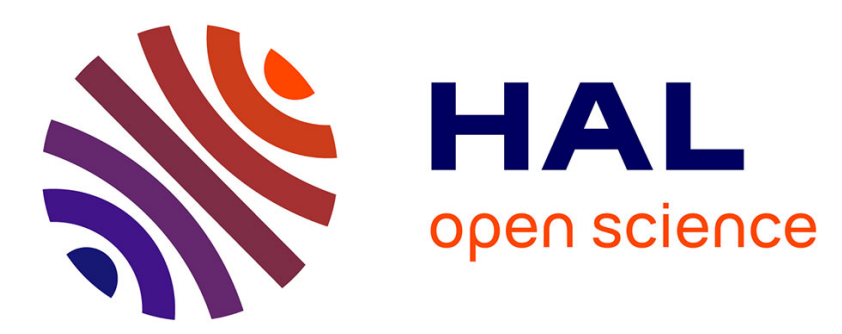

\title{
Antenna Modeling Based on a Multiple Spherical Wave Expansion Method: Application to an Antenna Array
}

Mohammed Serhir, Philippe Besnier, M'Hamed Drissi

\section{To cite this version:}

Mohammed Serhir, Philippe Besnier, M'Hamed Drissi. Antenna Modeling Based on a Multiple Spherical Wave Expansion Method: Application to an Antenna Array. IEEE Transactions on Antennas and Propagation, 2010, 58 (1), pp.51. 10.1109/TAP.2009.2036284 . hal-00553812

\section{HAL Id: hal-00553812 https://hal.science/hal-00553812}

Submitted on 9 Jan 2011

HAL is a multi-disciplinary open access archive for the deposit and dissemination of scientific research documents, whether they are published or not. The documents may come from teaching and research institutions in France or abroad, or from public or private research centers.
L'archive ouverte pluridisciplinaire HAL, est destinée au dépôt et à la diffusion de documents scientifiques de niveau recherche, publiés ou non, émanant des établissements d'enseignement et de recherche français ou étrangers, des laboratoires publics ou privés. 


\title{
Antenna Modeling based on a Multiple Spherical Wave Expansion Method: Application to an Antenna Array
}

\author{
Mohammed Serhir, Philippe Besnier Member, IEEE, and M'hamed Drissi, Senior Member, IEEE
}

\begin{abstract}
In this paper, a method to derive an equivalent radiation source for planar antennas is presented. This method is based on spherical near-field (NF) data (measured or computed) to ascertain an equivalent set of infinitesimal dipoles placed over the main antenna aperture. These produce the same antenna radiation field, both inside and outside the minimum sphere enclosing the antenna. A spherical wave expansion (SWE) of the NF data is written in terms of infinitesimal dipoles using a transition matrix. This matrix expresses the linear relations between the transmission coefficients of the antenna and the transmission coefficients of each dipole. The antenna a priori information are used to set the spatial distribution of the equivalent dipoles. The translational and rotational addition theorems are exploited to derive the transmission coefficients of the dipoles. Once the excitation of each dipole is known, the field at any aspect angle and distance from the antenna is rapidly calculated. Computations with EM simulation data of an antenna array illustrate the reliability of the method.
\end{abstract}

Index Terms-Antenna modeling, spherical wave expansion, translational and rotational addition theorems

\section{INTRODUCTION}

$\mathrm{T}$ HE antenna modeling in its operating environment is still a challenge using full-wave solutions. Indeed, the cost of such solutions grows exponentially with the size and the complexity of the problem. To cope with these difficulties, a two-steps hybrid approach is proposed. The antenna under test (AUT) is separately characterized and a simple equivalent model is defined. This comprises point sources that emulate the antenna radiation everywhere.

At the same time, when large antennas (antenna array) are hardly characterized by full-wave solutions, the measurement alternative can be of great interest, especially, the spherical multi-probe near-field technique [1]. This technique enables real-time 3D complex characterization all over the sphere surrounding the antenna provided the Nyquist sampling criterion is satisfied [2]-[3]. The antenna measured near field data is used then to define an equivalent model that allows the knowledge of the radiated EM field both inside and outside the minimum sphere enclosing the antenna. Thereafter, the

Manuscript received September 28, 2007, revised November 2008. The authors are with the Institute of Electronics and Telecommunications of Rennes (I.E.T.R), I.N.S.A. de Rennes, 35043 Rennes Cedex, France (email : mohammed_serhir@yahoo.fr). antenna equivalent model is incorporated into an existing EM algorithm to simulate multiple interaction scenes.

However, typical constraints arise, while modeling an antenna by an equivalent current/charge distribution. First, the equivalent model must reproduce the same radiated field as the antenna in near and far field regions. Secondly, the equivalent current/charge constituting the equivalent model must be consistent with the actual antenna current/charge distribution. Thirdly, we have to define a minimization technique that allows an efficient and well-conditioned identification process.

In the literature, different approaches were adopted. In [4][9] an electric field integral equation method (EFIE) is developed to relate the measured electric field to equivalent magnetic /electric current on the antenna aperture. The EFIE is solved using the conjugate gradient method. Equivalent currents are written as linear combinations of two-dimensional pulse basis functions, which can be approximated by Hertzian dipoles distributed over any enclosing arbitrary surface. This method has been investigated for near field to far field transformation, inverse electromagnetic radiation applications, antenna diagnosis. In [10] different strategy for antenna diagnosis is proposed. It expresses the relation between the plane wave expansion and the spherical wave expansion, which permits the calculation of the aperture field accurately.

In [11]-[13] evolutionary algorithms were introduced. Using Genetic Algorithm (GA) technique, the AUT is substituted by a set of infinitesimal dipoles. These are distributed inside the volume enclosing the antenna. The choice of infinitesimal dipole sources is justified by the simplicity of its implementation in any EM code, and the GA shows its ability to determine the type (electric or magnetic), the position, the orientation and the excitation of each dipole. The GA shows some difficulties to determine the optimal solution, when dealing with a large number of dipoles, including convergence issues and prohibitive CPU computing time, while increasing the antenna size. In [14] quantum particle swarm optimization algorithm has been used to define a set of equivalent dipoles for antenna modeling. This method is based on an expansive iterative process, which depends on $7 \mathrm{xN}$ degrees of freedom, where $\mathrm{N}$ is the number of dipoles.

In a previous paper [15] the authors have introduced a modeling technique depending on $6 \mathrm{xN}$ unknowns, where $\mathrm{N}$ is the number of current sources. It consists in the substitution of 
the antenna with a set of infinitesimal dipoles distributed over the minimum sphere circumscribing the antenna. These equivalent dipoles distribution is well adapted to build up a well-conditioned matrix system but is not convenient to provide the near-field within the minimum sphere for planar antennas for example.

In the present paper, an improvement of the analysis presented in [15] is proposed. By exploiting the antenna $a$ priori information, the equivalent dipoles are placed over the main radiating sources and the spherical wave expansion (SWE) of the antenna radiated field is rewritten, respecting a convenient change of basis, by means of small number of equivalent electric and magnetic dipoles that allow the knowledge of the near-field information inside the minimum sphere circumscribing the antenna. Once the antenna equivalent model of is achieved, the EM field can be rapidly calculated at any distances in a straightforward manner (faster than the SWE), using the free space green's function.

The paper is organized as follows. Section II describes the theoretical procedure of the method. Section III presents the application process of the method, regarding the equivalent dipoles locations, to an antenna-array. Finally, concluding remarks summarizing the potential of this method are provided in Section IV.

All theoretical results are expressed in the S.I. rationalized system with $e^{-j \omega t}$ time dependence.

\section{Formulations AND ThEORETICAL DEVELOPMENTS}

The complete formulation is given in [15]. We therefore, recall only the essential parts of this development, with a particular attention to the modifications introduced by the new arrangement of elementary source locations.

Here, a current source is defined as a combination of 4 tangential uncoupled and co-localized infinitesimal dipoles: 2 electric and 2 magnetic. We attach a local coordinate system $\left(o_{i}, \vec{x}_{i}, \vec{y}_{i}, \vec{z}_{i}\right)_{1 \leq i \leq L_{\text {source }}}$ to each current source, where the origin $o_{i}\left(r_{i}, \theta_{i}, \varphi_{i}\right)$ coincides with the position of the $\mathrm{i}^{\text {th }}$ source. Explicitly, we intend to rewrite the spherical wave expansion (SWE) expressed in $(o, \vec{x}, \vec{y}, \vec{z})$ by means of multiple and local SWE that are expressed in $\left(o_{i}, \overrightarrow{x_{i}}, \vec{y}_{i}, \vec{z}_{i}\right) \quad 1 \leq i \leq L_{\text {source }}$

Our purpose is to emulate the electromagnetic field radiated from an antenna by a set of current sources ( $L_{\text {saurce }}$ ) placed over the antenna main radiating surface (Fig. 1). Consequently, the field inside the minimum sphere is reached and the number of equivalent sources required to emulate accurately the antenna E-field, is reduced, while preserving the whole SWE information.

\section{A. Spherical wave expansion}

In source free region outside the minimum sphere of radius $r_{\min }$ circumscribing the antenna, the spherical wave expansion (SWE) of the radiated electric field $\vec{E}_{A U T}$ in spherical coordinates system is expressed in terms of truncated series of spherical vector wave functions [16] as

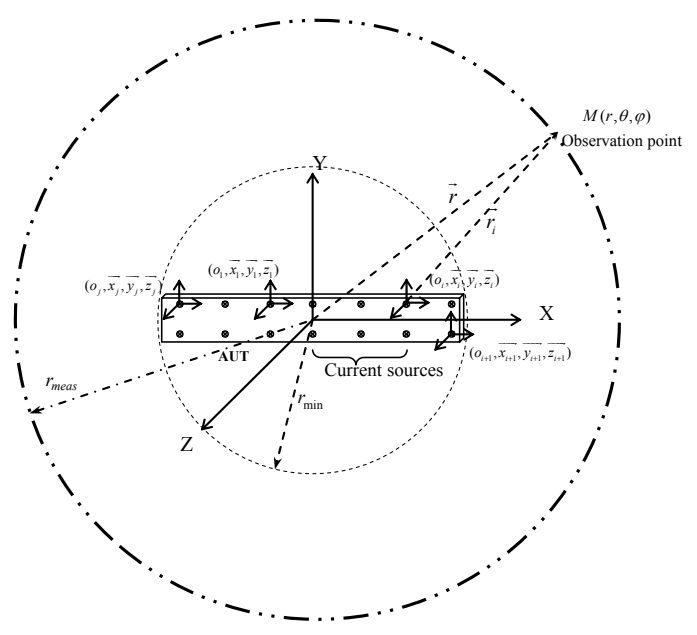

Fig. 1. Measurement sphere of radius $r_{\text {meas }}$, AUT minimum sphere and current sources configuration

$$
\vec{E}_{A U T}(\vec{r})=\frac{k}{\sqrt{\eta}} \sum_{j=1}^{J_{\max }} Q_{j} \vec{F}_{j}^{(3)}(\vec{r})
$$

where, $Q_{j}$ are the transmission coefficients, and $\vec{F}_{j}^{(3)}(\vec{r})$ are the power-normalized spherical vector wave functions. The truncation number $J_{\max }=2\left(N_{t r}\left(N_{t r}+2\right)\right)$ with, $N_{t r}=\left[k r_{\text {min }}\right]+10$ depends mainly on the antenna dimensions and the operating frequency [16]. The $Q_{j}$ are determined from the knowledge of the tangential components of either $\vec{E}$ or $\vec{H}$ on the measurement sphere. In [16], the $Q_{j}$ coefficients are determined using the orthogonal properties of spherical wave functions $\vec{F}_{j}^{(3)}(\vec{r})$. Once these coefficients are known, the field outside the minimum sphere is completely characterized by (1).

The SWE of the field radiated by an infinitesimal x-directed electric dipole $\overrightarrow{E_{i}^{e, x}}$ placed at the origin of $\left(o_{i}, \vec{x}_{i}, \vec{y}_{i}, \overrightarrow{z_{i}}\right)$ is expressed in the spherical coordinate system $\vec{r}_{i}\left(r_{i}, \theta_{i}, \varphi_{i}\right)$ associated with $\left(o_{i}, \vec{x}_{i}, \vec{y}_{i}, \vec{z}_{i}\right)$ as

$$
\overrightarrow{E_{\mathrm{i}}^{e, x}}\left(\overrightarrow{r_{i}}\right)=\frac{k}{\sqrt{\eta}} \frac{\sqrt{2}}{2} \alpha_{\mathrm{i}}^{e, x}\left(\vec{F}_{2}^{(3)}\left(\vec{r}_{i}\right)-\vec{F}_{6}^{(3)}\left(\vec{r}_{i}\right)\right)
$$

$$
\text { with } \quad \alpha_{i}^{e, x}=-\frac{k}{\sqrt{6 \eta \pi}} I_{i}^{e, x} l .
$$

Expressions for $\mathrm{z}$ and $\mathrm{y}$ - directed dipoles either electric or magnetic ones are provided in [15].

In the case of a planar antenna, the antenna equivalent dipoles are distributed over the antenna main surface and only the tangential dipoles are considered. Therefore, each current source $\left(i^{\text {th }}\right)$ is characterized by 4 transmission coefficients $\alpha_{i}^{e, x}, \alpha_{i}^{m, x}, \alpha_{i}^{e, y}, \alpha_{i}^{m, y}$. We refer to the $\mathrm{i}^{\text {th }}$ current source by the row vector $S_{i}=\left[\alpha_{i}^{e, x}, \alpha_{i}^{m, x}, \alpha_{i}^{e, y}, \alpha_{i}^{m, y}\right]$.

\section{B. The problem synthesis}

Using translational and rotational addition theorems [16]- 


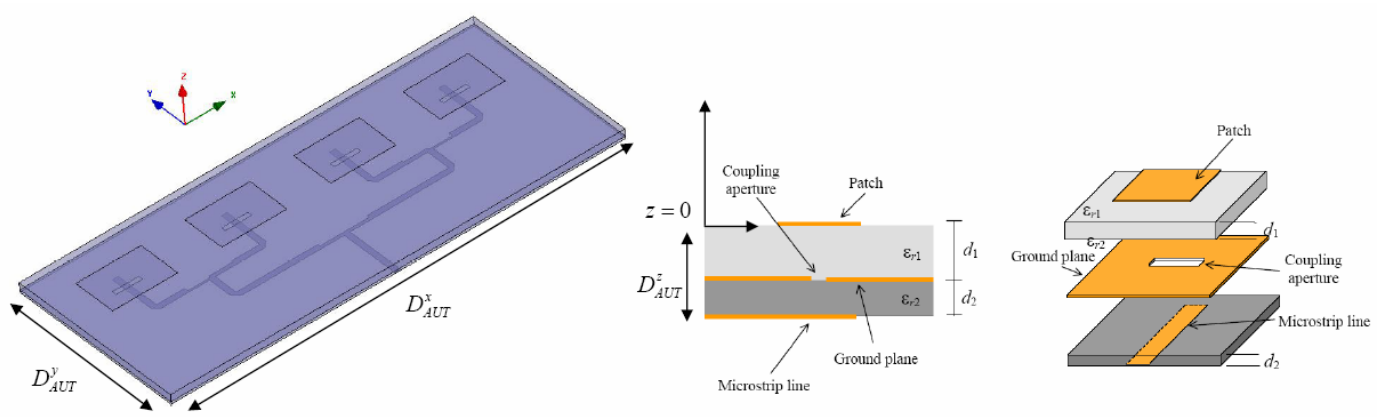

Fig. 2. 3D view of the microstripe array structure. Dielectric stratification (middle) and exploded view of the structure (right).

[17][18], we express the field radiated by the $\mathrm{i}^{\text {th }}$ current source in the antenna coordinate system $(o, \vec{x}, \vec{y}, \vec{z})$. Hence, the row vector $S_{i}$ is expressed in $(o, \vec{x}, \vec{y}, \vec{z})$ by

$$
\left[M_{i, j}\right]_{j=1 . . J_{\max }}=\left[\alpha_{i}^{e, x} A_{j, i}^{e, x}, \alpha_{i}^{m, x} A_{j, i}^{m, x}, \alpha_{i}^{e, y} A_{j, i}^{e, y}, \alpha_{i}^{m, y} A_{j, i}^{m, y}\right]_{j=1 . . J_{\max }}
$$

where the coefficients $A_{j, i}^{e, x}, A_{j, i}^{m, x}, A_{j, i}^{e, y}, A_{j, i}^{m, y}$ are developed in the Appendix of [15]. Let define $\mathcal{A}_{j, i}=\left[A_{j, i}^{e, x}, A_{j, i}^{m, x}, A_{j, i}^{e, y}, A_{j, i}^{m, y}\right]$.

The superposition of the fields radiated by all equivalent dipoles $\left(\vec{E}_{\mathrm{mod}}\right)$ is written in $(o, \vec{x}, \vec{y}, \vec{z})$ as

$$
\begin{aligned}
\vec{E}_{\bmod }(\vec{r}) & =\frac{k}{\sqrt{\eta}} \sum_{i=1}^{L_{\text {source }}} \sum_{j=1}^{J_{\max }}\left(\begin{array}{l}
\alpha_{i}^{e, x} A_{j, i}^{e, x}+\alpha_{i}^{m, x} A_{j, i}^{m, x} \\
+\alpha_{i}^{e, y} A_{j, i}^{e, y}+\alpha_{i}^{m, y} A_{j, i}^{m, y}
\end{array}\right) \vec{F}_{j}^{(3)}(\vec{r}) \\
& =\frac{k}{\sqrt{\eta}} \sum_{j=1}^{J_{\max }} \sum_{i=1}^{L_{\text {source }}}\left[\mathfrak{\uparrow}_{j, i}\right] \cdot S_{i}{ }^{\mathrm{T}} \cdot \vec{F}_{j}^{(3)}(\vec{r})
\end{aligned}
$$

Identifying $\vec{E}_{A U T}$ with $\vec{E}_{\text {mod }}$, and from (1) and (4) we get

$$
\sum_{j=1}^{J_{\max }} Q_{j} \vec{F}_{j}^{(3)}(\vec{r})=\sum_{j=1}^{J_{\max }} \sum_{i=1}^{L_{\text {source }}}\left[\boldsymbol{\vartheta}_{j, i}\right] \cdot S_{i}^{\mathrm{T}} \cdot \vec{F}_{j}^{(3)}(\vec{r})
$$

Due to the orthogonal properties of $\vec{F}_{j}^{(3)}$, the transmission coefficients of both sides of (5) have to be equal.

Let denote $\boldsymbol{A}=\left(\boldsymbol{f}_{j, i}\right)_{\substack{1 \leq i \leq L_{\text {source }} \\ 1 \leq j \leq J_{\max }}}, \quad X=\left[\begin{array}{ll}S_{1} \ldots & S_{L_{\text {source }}}\end{array}\right]^{\mathrm{T}}$ and $\mathcal{Q}=\left[\begin{array}{ll}Q_{1} \ldots & Q_{j_{\max }}\end{array}\right]^{\mathrm{T}}$, where the superscript ${ }^{\mathrm{T}}$, expresses the matrix transpose. Equation (5) becomes

$$
\mathbb{A} . X=\mathcal{Q} \text {. }
$$

Made up of $4 \times L_{\text {source }}$ columns and $J_{\max }$ lines, $\mathcal{A}$ is the transition matrix, which expresses linear relations between the transmission coefficients of current sources $(X)$ and the transmission coefficients $Q$ of the actual antenna. To solve (6), we use the least square (LSQR) code of MatLab to determine the $4 \times L_{\text {source }}$ unknowns composing the row vector $x$.

\section{RESULTS}

\section{A. Example of equivalent models of an antenna array}

In this section, we report a numerical example by simulation of a linear microstrip array of aperture-coupled

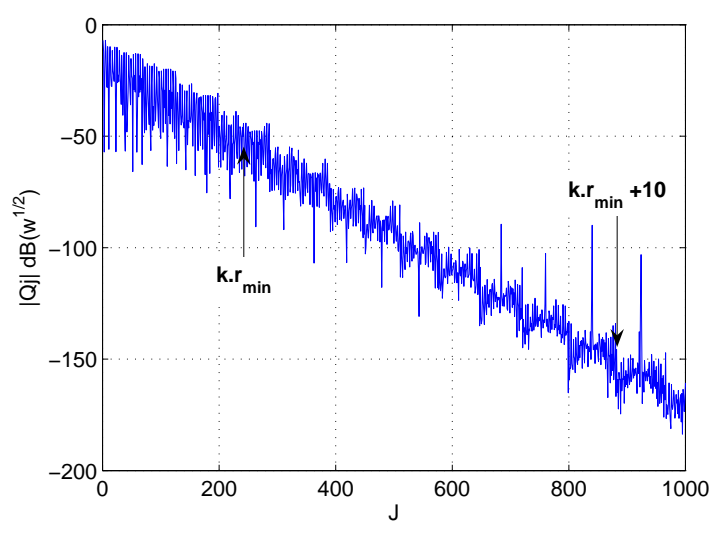

Fig. 3. Magnitude of the antenna array transmission coefficients

patches operating at the frequency $5.82 \mathrm{GHz}$. The antenna dimensions are $D_{A U T}^{x}=0.1464 m(2.84 \lambda)$ along the x-axis, $D_{A U T}^{y}=0.06 \mathrm{~m}(1.16 \lambda)$ along the y-axis, and $D_{A U T}^{z}=0.0031 \mathrm{~m}$ along the z-axis with $r_{\min }=0.085 m(1.65 \lambda)$ (Fig.2). The antenna is composed of a linear array of 4 rectangular patches printed on a dielectric support. Each patch is aperture-coupled to the microstrip line. A common ground plane separates the radiating part from the microstrip feeding network (Fig.2). The antenna is issued from Antenna Center of Excellence benchmarking data base, where the detailed description is provided in [21].

The antenna equivalent model construction is based on near field data obtained through the electromagnetic simulation of the antenna in a 3D simulator that uses the finite elements method (FEM). We recover at the distance of $r_{\text {meas }}=15 \lambda(\approx 0.75 \mathrm{~m})$, the amplitude and phase of both E-field components $E_{\theta}$ and $E_{\varphi}$ over a spherical surface $(0 \leq \theta \leq \pi$, $0 \leq \varphi \leq 2 \pi)$, with the angular resolution of $\Delta \theta=\Delta \varphi=5 \mathrm{deg}$. Based on these data, we assess the antenna transmission coefficients that are presented in Fig.3. The SWE is truncated at $N_{t r}=\left[k r_{\text {min }}\right]+10=20$, so $J_{\max }=2 N_{t r}\left(N_{t r}+2\right)=880$ transmission coefficients $Q_{j}$ is considered. 

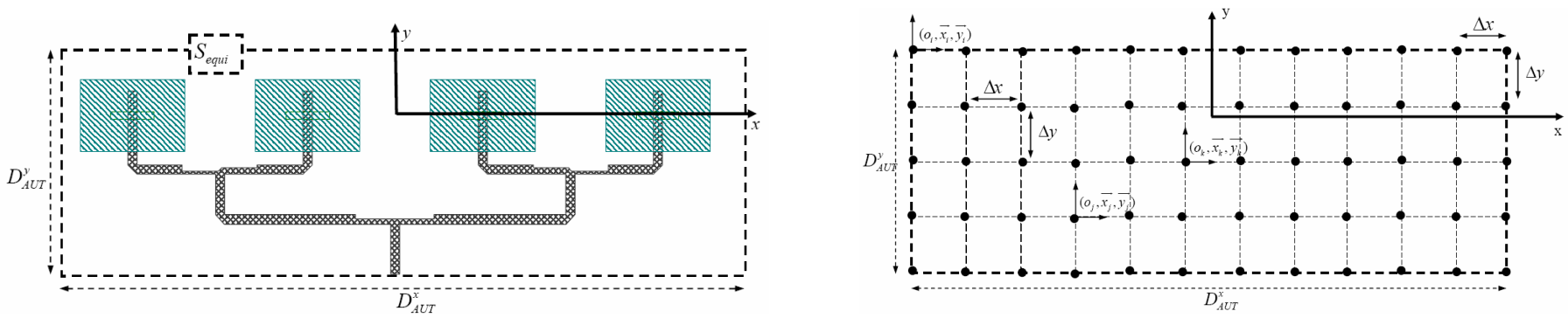

Fig. 4. The description of the equivalent current sources distribution over the antenna surface. The dashed rectangle represents the antenna equivalent surface. (right) The black points represent the equivalent current sources distributed regularly over the antenna equivalent surface respecting the criterion $\Delta x=\Delta y=0.25 \lambda$.

The antenna being planar, we have chosen the natural way to place the current sources (rectangular grid). The antenna is seen as the rectangular surface

$$
S_{\text {equi }}=(x, y, z) \in\{-0.073 m \leq x \leq 0.073 m,-0.042 m \leq y \leq 0.017 m, z=0\}
$$

over which, the equivalent dipoles are uniformly distributed (Fig.4). The antenna equivalent model is associated with a spacing criterion $\Delta x$ and $\Delta y$ between the current sources. Each criterion results in a number of current sources $L_{\text {saurce }}=\left[\left(D_{A U T}^{x} / \Delta x\right)+1\right] \cdot\left[\left(D_{A U T}^{y} / \Delta y\right)+1\right]$ where $[X]$ means the integer part of $x$.

We attach a local coordinate system $\left(o_{i}, \vec{x}_{i}, \overrightarrow{y_{i}}\right)$ to each current source, we determine the corresponding transition matrix $f$ and we solve (6) to calculate the excitation of each dipole. Then, the E-field radiated from the equivalent dipoles $E^{\text {mod }}$ is calculated at different spherical observation domains, near the minimum sphere and in the far field regions and compared with the actual antenna radiation pattern $E^{a c t}$ using the following error function:

$R M S_{E}=\sqrt{\frac{\sum_{\theta, \varphi}\left|E^{a c t}(\theta, \varphi)-E^{\bmod }(\theta, \varphi)\right|^{2}}{\sum_{\theta, \varphi}\left|E^{a c t}(\theta, \varphi)\right|^{2}}}$, where $E=E_{\theta}, E_{\varphi}$

Comparisons between the antenna radiation pattern (Finite Elements Method) and the radiation pattern of the equivalent dipoles in near and far field regions are shown in Fig. 5 for the $\Delta x=\Delta y=0.25 \lambda$ and $\Delta x=\Delta y \simeq 0.4 \lambda$. As it can be seen, the figures show good agreements and the equivalent models radiation pattern fit very well with the one resulted from the spherical wave expansion method. In Fig. 6, the error function is presented as a function of the distance from the antenna. It is seen that the equivalent model corresponding to $\Delta x=\Delta y \simeq 0.25 \lambda$ results in $0.23 \% \leq R M S_{E_{o}} \leq 0.29 \%$ and $0.16 \% \leq R M S_{E \varphi} \leq 0.31 \%$. Using the criterion $\Delta x=\Delta y=0.4 \lambda$, the equivalent model results in $0.77 \% \leq R M S_{E_{\theta}} \leq 0.88 \%$ and $0.75 \% \leq R M S_{E \varphi} \leq 1 \%$. For a smaller spacing $\Delta x=\Delta y=0.2 \lambda$, the accuracy of the antenna equivalent model is improved $\left(0.04 \% \leq R M S_{E_{\theta}} \leq 0.1 \%, 0.08 \% \leq R M S_{E \varphi} \leq 0.17 \%\right)$ but the number of equivalent sources increases $(\Delta x=\Delta y=0.2 \lambda$ corresponds to 90 current sources). These equivalent models reproduce accurately the antenna radiation everywhere outside the minimum sphere $(2 \lambda \leq$ distance $\leq 40 \lambda)$.

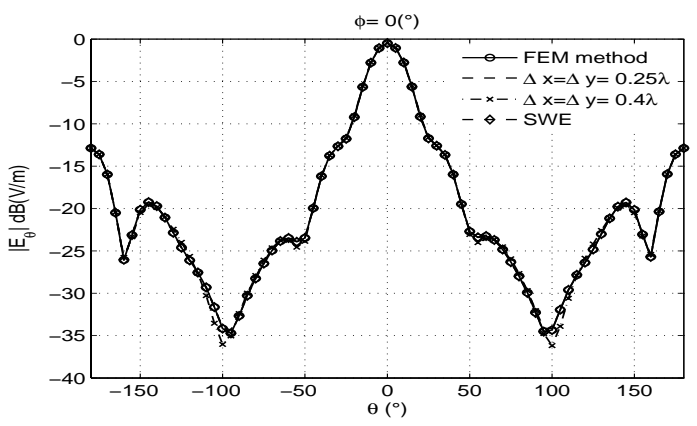

(a)

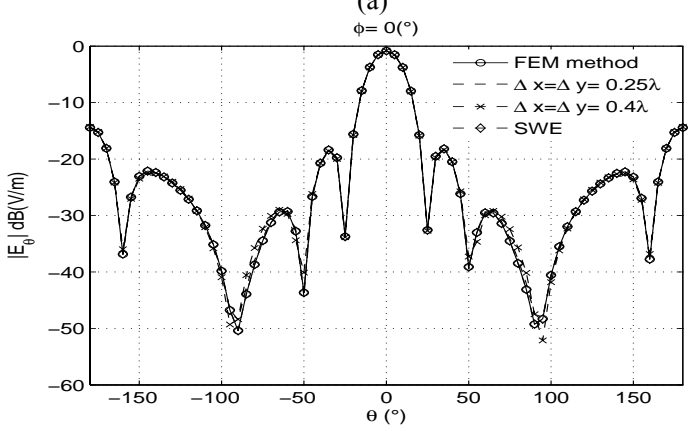

(b)

Fig .5 At the plane $\phi=0^{\circ}$, (a) Near-field observed at the distance of $4 \lambda$. (b) Far-field radiation pattern. The actual field (FEM) (o-marker line), the field resulted from the SWE ( $\square$-marker line), and the field radiated from the equivalent models associated with $\Delta \mathrm{x}=\Delta \mathrm{y}=0.25 \lambda$ and $0.4 \lambda$.

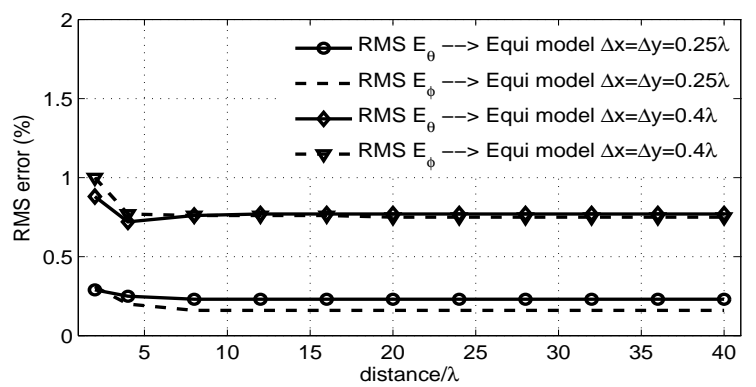

Fig. 6 The error function RMSE versus the distance for $\Delta x=\Delta y=0.25 \lambda$ and $0.4 \lambda$.associated equivalent models.

In conclusion, the spacing between the current sources is related to the accuracy of the equivalent model. Consequently, the choice of the spacing between the equivalent current sources $\Delta x, \Delta y$ may be based on a targeted error budget for a given application. From a numerical point of view, the spacing between sources has a direct effect over the condition of the matrix $A$ in (6). Using a large spacing $\Delta x=\Delta y \geq 0.5 \lambda$ the matrix $A$ is well-conditioned $(\Delta x=\Delta y=0.6 \lambda$ corresponds 
to $\operatorname{cond}(\boldsymbol{A})=3.17)$, however, the accuracy of the equivalent model is decreased $\left(11 \% \leq R M S_{E_{\theta}} \leq 14 \%\right.$ and $\left.4.2 \% \leq R M S_{E \varphi} \leq 7.4 \%\right)$. Decreasing the spacing $\Delta x$ and $\Delta y$, the accuracy of the equivalent model is improved but the matrix of becomes illconditioned, where $\operatorname{cond}(\mathcal{A})=6.574 .10^{5}$ and $\operatorname{cond}(\mathcal{(})=122$ for $\Delta x=\Delta y \simeq 0.25 \lambda$ and $\Delta x=\Delta y \simeq 0.4 \lambda$ respectively. As a matter of fact, we have to compromise between the accuracy and the conditioning of $\AA$.

Inside the minimum sphere, over the plane $\{-0.075 m \leq x \leq 0.075 m,-0.045 m \leq y \leq 0.02 m, z=0.02 m\}$ located at the distance $z=0.02 m(0.4 \lambda)$ from the antenna, the Ey-field component estimated by the 3D simulation software (Finite elements method) and the Ey-field radiated by the equivalent dipoles are presented in Fig.8. A good agreement is observed, between the actual antenna radiation pattern and the equivalent model radiation pattern. For the near-field presented in Fig.8, the error function (Ey component) reaches $1.42 \%$.

As presented in Fig.9, the equivalent models ( $\Delta x=\Delta y=0.4 \lambda$ or $0.25 \lambda$ ) provide a good approximation of the field inside the minimum sphere for distances greater than $0.3 \lambda$ from the antenna. Otherwise, the field in the vicinity of the antenna (distance $\prec 0.2 \lambda$ ) is mainly reactive and the information of the evanescent modes are not available at the measurement distance $\left(r_{\text {meas }}=15 \lambda\right)$. Consequently, the equivalent models presents some discrepancies $\left(R M S_{E} \geq 13 \%(\Delta x=\Delta y=0.25 \lambda)\right)$, while trying to reproduce the Efield in the vicinity of the antenna ( distance $=0.2 \lambda$ ). At the distance of $0.3 \lambda, R M S_{E_{y}}$ takes the values $4.32 \%$ and $7.28 \%$ for the equivalent models associated with $\Delta x=\Delta y \simeq 0.25 \lambda$ and $\Delta x=\Delta y \simeq 0.4 \lambda$ respectively.

The aim of the examples presented here, is to show the feasibility of the modeling method for the case of a finite open surface (equivalence principle for an open surface) and to figure out how to compromise between the accuracy and the number of equivalent sources (simplicity of the equivalent model). The flexibility of the presented modeling methodology is outlined.

Exploiting the a priori information concerning the antenna geometry, we have defined an equivalent model, which reproduces accurately the antenna radiation pattern. We have established that this modeling technique is able to define different kind of equivalent models where the choice of the spacing between equivalent current sources (complexity) depends on the desired accuracy, so, on the application for which the equivalent model is intended.

\section{B. The reduction of the number of equivalent model dipoles}

Solving (6), we determine the row vectors $S_{i}=\left[\alpha_{i}^{e, x}, \alpha_{i}^{m, x}, \alpha_{i}^{e, y}, \alpha_{i}^{m, y}\right]$ for all current sources. By means of the normalized row vector $S_{i_{-} \text {nor }}$ expressed as

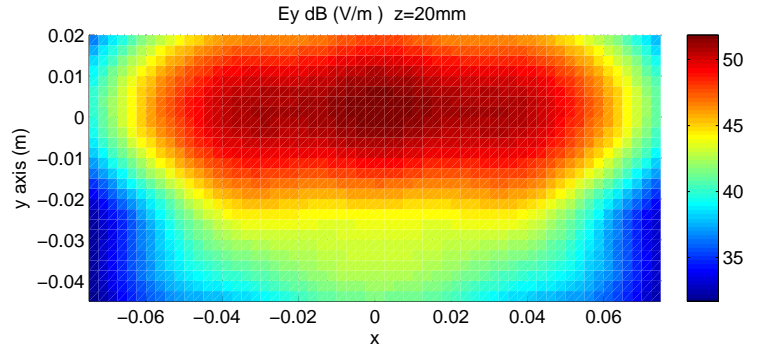

(a)

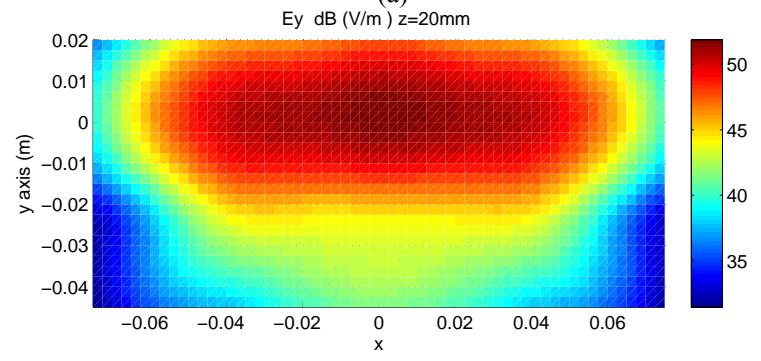

(b)

Fig .8 The aperture field $\mathrm{Ey}(\mathrm{dB} \mathrm{V} / \mathrm{m})$ at the plane $\mathrm{z}=0.02 \mathrm{~m}(0.4 \lambda)$ from the AUT: (a) Resulted from the FEM simulation software. (b) Produced by the equivalent model associated to $\Delta \mathrm{x}=\Delta \mathrm{y}=0.25 \lambda$.

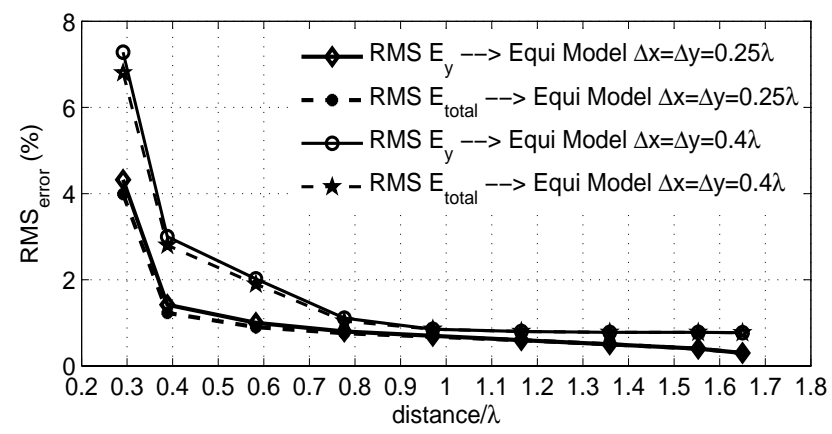

Fig.9 The error function RMSE evaluated inside the minimum sphere versus the distance for $\Delta \mathrm{x}=\Delta \mathrm{y}=0.25 \lambda$ and $0.4 \lambda$. associated equivalent models.

$S_{i_{-} \text {nor }}=\frac{1}{2 P_{i}}\left[\left|\alpha_{i}^{e, x}\right|^{2},\left|\alpha_{i}^{m, x}\right|^{2},\left|\alpha_{i}^{e, y}\right|^{2},\left|\alpha_{i}^{m, y}\right|^{2}\right]$

where $P_{i}=\frac{1}{2}\left(\left|\alpha_{i}^{e, x}\right|^{2}+\left|\alpha_{i}^{m, x}\right|^{2}+\left|\alpha_{i}^{e, y}\right|^{2}+\left|\alpha_{i}^{m, y}\right|^{2}\right)$

we evaluate the power contribution of each dipole constituting the current source. The elements of $S_{i_{\text {nnor }}}$ correspond to the power contribution of each dipole in the current source radiation power $P_{i}$.

By a proper choice of the threshold $T h$, we remove the insignificant dipoles, so that the number of the dipoles constituting the equivalent model is reduced. An iterative process is carried out.

Firstly, we choose a spacing criterion between the equivalent current sources $(\Delta x=\Delta y=0.4 \lambda$ is a good compromise between the accuracy and the number of equivalent current sources). Secondly, respecting a uniform distribution, we determine the spatial position of the current sources over the planar antenna main radiating surface. Thirdly, we solve (6) taking into account all the dipoles and we evaluate the row vectors $\left\{S_{i_{-} \text {nor }}\right.$ for $\left.i \in \llbracket 1 . . L_{\text {source }} \|\right\}$ so that one can eliminate the dipoles whose corresponding terms in 


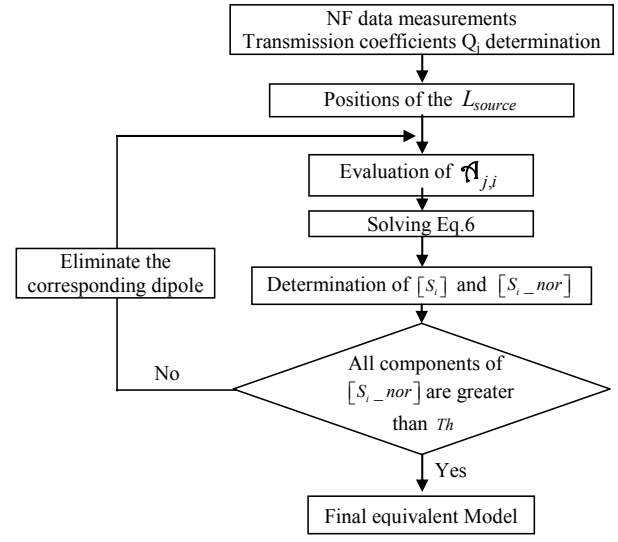

Fig. 10. The description of the reduction of the number of equivalent model dipoles procedure.

$S_{i \text { nor }}$ are less than $T h$. Finally, a new solution of (6) is performed providing the new configuration (without the insignificant dipoles). This procedure is pursued as long as non-significant dipoles are detected. The flowchart describing the complete proposed modeling procedure is outlined in Fig. 10.

Considering the antenna equivalent model based on $\Delta x=\Delta y \simeq 0.25 \lambda$. The reduced equivalent models configurations corresponding to different thresholds $T h$ are presented in Table I. Then, the E-field radiated from these reduced equivalent models is compared with the actual E-field estimated by FEM at different observation distances $2 \lambda \leq$ distance $\leq 40 \lambda$.

From Fig. 11, where we present $R M S_{E}$ as function of the distance, we observe that the same level of accuracy is maintained after the reduction procedure for $T h=2 \%, 4 \%$ $\left(0.52 \% \leq R M S_{E_{\theta}}, R M S_{E_{\varphi}} \leq 0.73 \%\right)$, while reducing considerably the number of the equivalent dipoles (240 dipoles for $T h=0 \%$ to 126 dipoles for $T h=4 \%$ ).

Since the actual radiating currents are due to the patches, which are aperture-coupled to the microstrip line, the actual current sources depends on the geometry of the apertures and the patches. The apertures are aligned with the $\mathrm{x}$-axis, so the antenna equivalent dipoles must include $x$-directed magnetic dipoles. On the other hand, the rectangular patches include $\mathrm{x}$ directed and y-directed electric dipoles.

As presented in TABLE I, after the reduction procedure, the dipoles types constituting the reduced equivalent models are: $\mathrm{y}$-directed, $\mathrm{x}$-directed electric dipoles, and $\mathrm{x}$-directed magnetic dipoles. These dipoles are consistent, from a qualitative point of view, with the antenna actual current sources.

For the equivalent model based on $\Delta x=\Delta y \simeq 0.25 \lambda$, we converge toward a reduced configuration of the equivalent model (using $T h=4 \%$ ) while fitting accurately $(99 \%)$ the actual antenna radiation pattern. This threshold allows us to reproduce accurately the radiated E-field as the actual antenna using only 126 dipoles (126 coefficients). In other words, the use of a priori information concerning the antenna geometry to define the multiple-SWE can be of great interest since it
TABLE I

EQUIVALENT MODELS AFTER THE REDUCTION PROCEDURE CASE: $\Delta x=\Delta y=0.25 \lambda$ FOR $T h=2,4 \%$

\begin{tabular}{|c|c|c|c|c|}
\hline \multicolumn{2}{|c|}{$\begin{array}{c}\text { Number of current } \\
\text { sources }\end{array}$} & $\begin{array}{c}\text { WITHOUT } \\
\text { FILTRING }\end{array}$ & $T h=2 \%$ & $T h=4 \%$ \\
\hline \multirow{4}{*}{$\begin{array}{c}L_{\text {source }}=60 \\
\text { current } \\
\text { sources }\end{array}$} & Total & $\begin{array}{c}240 \\
\text { dipoles }\end{array}$ & $\begin{array}{c}184 \\
\text { dipoles }\end{array}$ & $\begin{array}{c}126 \\
\text { dipoles }\end{array}$ \\
\cline { 2 - 5 } & dipole $^{e, y}$ & 60 & 60 & 50 \\
\cline { 2 - 5 } & dipole $^{e, x}$ & 60 & 36 & 33 \\
\cline { 2 - 5 } & dipole $^{m, y}$ & 60 & 44 & 5 \\
\cline { 2 - 5 } & dipole $^{m, x}$ & 60 & 44 & 38 \\
\hline
\end{tabular}

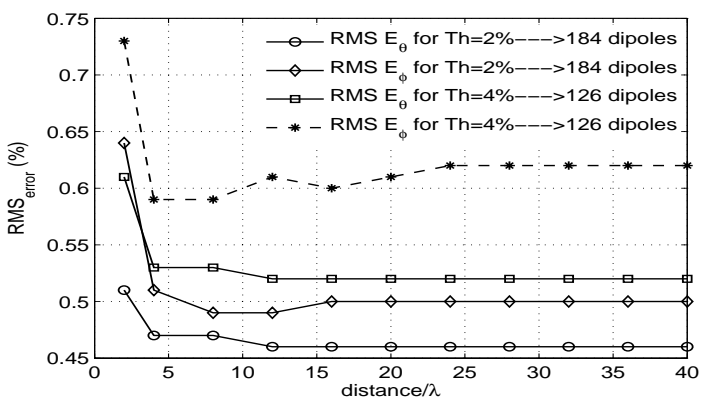

(a)

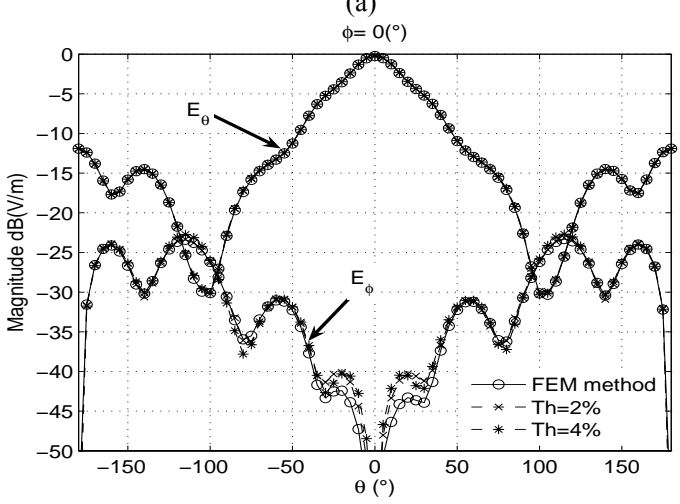

(b)

Fig.11 (a) The error function RMSE corresponding to the thresholds th=2 and $4 \%$ as a function of the distance resulting from the equivalent model associated with $\Delta \mathrm{x}=\Delta \mathrm{y}=0.25 \lambda$. (b) The near field observed at the distance $2 \lambda$ radiated from the simplified equivalent models.

permits to rewrite the transmission coefficients of the antenna in limited number of equivalent dipole excitations. The reduction procedure tends to cancel dipoles that do not contribute effectively in the radiation pattern.

Contrarily to the spherical wave expansion, the minimum number of transmission coefficients is related to the antenna dimensions. At least we should consider the truncation number $N_{t r}=\left[k r_{\min }\right]=10$, which leads to the use of $J_{\max }=2 N_{t r}\left(N_{t r}+2\right)=240$ transmission coefficients. The SWE is a very accurate way to describe the radiated field and the accuracy of the SWE cannot be questioned. Nonetheless, the SWE is valid outside the minimum sphere and the implementation of the SWE representation of the radiated field in an electromagnetic code is not trivial. Rewriting the transmission coefficients (SWE) in terms of equivalent dipoles, enhanced with the reduction procedure, can be a good way to express the whole information contained in the 
spherical wave coefficients in a limited number of dipole excitations.

The rearrangement of the SWE into a multiple SWE expressed in different local coordinate systems (using a priori information) is a time consuming procedure (translational and rotational addition theorems). Nevertheless, once the equivalent model is determined, the calculation of the radiation pattern in different distances from the antenna is faster than using the SWE. This is due to the fact that the number of equivalent dipoles is smaller than the number of the transmission coefficients.

\section{CONCLUSION}

A method for antenna modeling has been presented. This method is based on the substitution of the original antenna by a set of equivalent infinitesimal dipoles that reproduce the same radiating field. Since the equivalent model includes a set of dipoles placed over the main antenna radiating surface, the information given by these dipoles gives qualitative information concerning the origin of the radiation. Using the antenna a priori information, it has been shown that the number of infinitesimal dipoles can be significantly reduced.

The use of the transmission coefficients to elaborate the antenna equivalent model is interesting. This allows a straightforward matching procedure, where the whole antenna intrinsic information are contained in a finite number of complex values. Once the antenna equivalent model is defined, the electromagnetic radiation of the antenna can be calculated at any point both inside and outside (FF region) the minimum sphere except in the reactive region of the antenna. Also, the antenna a priori information concerning the AUT geometry have been used to express the multiple spherical wave expansion, which is of great interest since it has allowed the rewrite the SWE with a limited and reduced number of dipole excitations.

\section{REFERENCES}

[1] J. C. Bolomey, B. J. Cown, G. Fine, L. Jofre, M. Mostafavi, D. Picard, J. P. Estrada, P. G. Friederich, F. L. Cain, "Rapid near-field antenna testing via arrays of modulated scattering probes," IEEE Trans. Antennas Propagat., Vol. 36, pp. 804-814, June 1988.

[2] A. D. Yaghjian, "An overview of near-field antenna measurements," IEEE Trans. Antennas Propagat., Vol. AP-34, pp. 30-45, Jan. 1986.

[3] O. M. Bucci, C. Gennarelli, and C. Savarese, "Representation of electromagnetic fields over arbitrary surfaces by a finite and non redundant number of samples," IEEE Trans. Antennas Propagat., Vol. 46, pp. 351-359, Mar. 1998.

[4] P. Petre and T. K. Sarkar, "Planar near-field to far-field transformation using an equivalent magnetic current approach" IEEE Trans. Antennas Propagat., Vol. 40, pp. 1348-1356, Nov. 1992.

[5] P. Petre and T. K. Sarkar, "Planar near-field to far field transformation using an array of dipole probes" IEEE Trans. Antenna Propagat., Vol. 42, pp. 534-537, Apr. 1994.

[6] T. K. Sarkar and A. Taaghol, "Near-field to near/far-field transformation for arbitrary near-field geometry utilizing an equivalent electric current and MoM" IEEE Trans. Antenna Propagat., Vol. 47, pp. 566-573, Mar. 1999.

[7] F. Las-Heras and T. K. Sarkar, "A direct optimization approach for source reconstruction and NF-FF transformation using amplitude-only data," IEEE Transact. Antenna Propagat., Vol. 50, pp. 500-510, Apr. 2002 .
[8] F. Las-Heras, M. R. Pino, S. Loredo, Y. Alvarez and T. K. Sarkar, "Evaluating near-field radiation patterns of commercial antennas," IEEE Transact. Antenna Propagat., Vol. 54, pp. 2198-2207, Aug. 2006.

[9] Y. Alvarez, F. Las-Heras, and M. R. Pino, "Reconstruction of equivalent currents distribution over arbitrary three-dimensional surfaces based on integral equation algorithms," IEEE Transact. Antenna Propagat., Vol. 55, pp. 3460-3468, Dec. 2007.

[10] C. Cappellin, A. Frandsen and O. Breinbjerg, "On the relationship between the spherical wave expansion and the plane wave expansion for antenna diagnostics," in Proc. AMTA Europe symp. 2006.

[11] J. R. Regué, M. Ribo, J. M. Garrell, and A. Martin, "A genetic algorithm based method for source identification and far-field radiated emissions prediction from near-filed measurement for PCB characterization" IEEE Trans. Electromagn. Compat., Vol. 43, No. 4, pp. 536-542, Aug. 1996.

[12] J. R. Pérez and J. Basterrechea, "Antenna far-field pattern reconstruction using equivalent currents and genetic algorithms" Microwave Opt. Technol. Lett., Vol.42, No. 1, pp. 21-25, July 2004.

[13] T. S. Sijher and A.A. Kishk, "Antenna modeling by infinitesimal dipoles using genetic algorithms" Progress In Electromagnetics Research, PIER 52, pp. 225-254, 2005.

[14] Mikki, S.M.and Kishk, A.A "Theory and Applications of Infinitesimal Dipole Models for Computational Electromagnetics," IEEE Transact. Antenna Propagat., Vol. 55, pp.1325 - 1337, May. 2007.

[15] M. Serhir, P. Besnier and M.Drissi, "An accurate equivalent behavioral model of antenna radiation using a mode-matching technique based on spherical near field measurements," IEEE Transact. Antenna Propagat., Vol. 56, pp. 48-57, Jan. 2008.

[16] J. E. Hansen, Spherical Near-Field Antenna Measurements. London, U.K.: Peregrinus, 1988.

[17] A. R. Edmonds, Angular Momentum in Quantum Mechanics, 3rd ed. Princeton, NJ: Princeton Univ. Press, 1974.

[18] J. H. Bruning and Y. T. Lo, "Multiple scattering of EM waves by spheres, Part I - Multipole expansion and ray-optical solutions," IEEE Trans. Antennas Propagat. Vol. Ap-19, pp. 378-390, May 1971.

[19] Link:http://ace1.antennasvce.org/Dissemination/view/download?id file $=43$ page 103

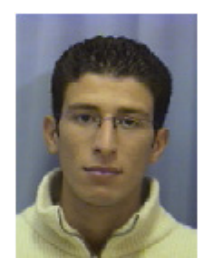

Mohammed Serhir was born January 8, 1981, in Casablanca, Morocco. He received the diplôme d'ingénieur degree from Ecole Mohammadia d'Ingénieurs (EMI), Rabat, Morocco in 2003 and the $\mathrm{Ph}$. D. degree in electronics from the National Institute of Applied Sciences at Rennes, INSA de Rennes in 2007. His research interests include spherical wave expansion technique, spherical near-field antenna measurements, and the development of numerical methods.

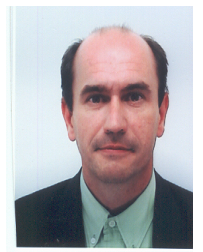

Philippe Besnier received the diplôme d'ingénieur degree from Ecole Universitaire d'Ingénieurs de Lille (EUDIL), Lille, France, in 1990 and the $\mathrm{Ph}$. D. degree in electronics from the university of Lille in 1993. He was with the Laboratory of Radio Propagation and Electronics, University of Lille, as a researcher at the Centre National de la Recherche Scientifique (CNRS) from 1994 to 1997. Since 2002, he has been with the Institute of Electronics and Telecommunications of Rennes, Rennes, France, where he is currently a researcher at CNRS heading EMC-related activities such as EMC modeling, electromagnetic topology, reverberation chambers, and near-field probing.

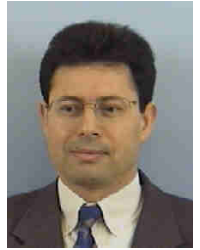

M'hamed Drissi (SM'90) received the Ph.D. degree in electronics from INSA of Rennes in 1989 and the HDR degree in 1997 from the University of Rennes 1, France. In 1991, he joined INSA of Rennes, where he is currently a full professor and director of research of INSA of Rennes. His research activities deal with the electromagnetic modelling and the design of antennas and the associated circuits, the electromagnetic compatibility of complex electronic systems, and the near-field characterization. His research interests include CAD of high-speed and MMIC circuits, neuronal modeling and simulation of high-speed interconnecting, and nonlinear circuits. 